\title{
On the Structure of Nonlinearities in Pose Graph SLAM
}

\author{
Heng Wang*, Gibson $\mathrm{Hu}^{\dagger}$, Shoudong Huang ${ }^{\dagger}$ and Gamini Dissanayake ${ }^{\dagger}$ \\ * College of Electronic Information and Control Engineering, \\ Beijing University of Technology, Beijing, China. Email:wangheng19801980@126.com \\ $\dagger$ Centre for Autonomous Systems, Faculty of Engineering and Information Technology, \\ University of Technology, Sydney, Australia. Email:\{Gibson.Hu, Shoudong.Huang, Gamini.Dissanayake\}@uts.edu.au
}

\begin{abstract}
Pose graphs have become an attractive representation for solving Simultaneous Localization and Mapping (SLAM) problems. In this paper, we analyze the structure of the nonlinearities in the 2D SLAM problem formulated as the optimizing of a pose graph. First, we prove that finding the optimal configuration of a very basic pose graph with 3 nodes (poses) and 3 edges (relative pose constraints) with spherical covariance matrices, which can be formulated as a six dimensional least squares optimization problem, is equivalent to solving a one dimensional optimization problem. Then we show that the same result can be extended to the optimizing of a pose graph with "two anchor nodes" where every edge is connecting to one of the two anchor nodes. Furthermore, we prove that the global minimum of the resulting one dimensional optimization problem must belong to a certain interval and there are at most 3 minima in that interval. Thus the globally optimal pose configuration of the pose graph can be obtained very easily through the bisection method and closed-form formulas.
\end{abstract}

\section{INTRODUCTION}

Recently, SLAM techniques based on pose graphs are becoming very popular because of their robustness and computational efficiency. Solving the SLAM problem using pose graph representation typically includes two stages. In the first stage (SLAM front-end), relative pose constraints are obtained (e.g. from odometry and/or scan matching) to construct a pose graph where each node is a robot pose and each edge is a relative pose constraint. In the second stage (SLAM backend), an optimization technique is applied to find the optimal pose configuration.

The concept of pose graph SLAM formulation has been introduced in [1]. Recent insights into the sparse structure of the SLAM problem have boosted the research in this direction. The work includes multi-level relaxation [2], stochastic gradient descent (SGD) [3], sparse Pose Adjustment (SPA) [4], preconditioned conjugate gradient and subgraph strategy [5]. A number of 3D pose graph SLAM algorithms have also been developed that can be applied to visual SLAM and SLAM with 3D laser data (http://openslam.org/).

The optimization problem involved in pose graph SLAM has a very high dimension because all the poses involved are variables. It is expected that such a high dimensional nonlinear optimization problem could have a very large number of local minima and general iteration based optimization algorithms could frequently be trapped into one of these local minima unless very good initial value is available. However, the results in [3][4][5] demonstrate that sometimes the algorithms can converge to good solution with poor or even zero initial values. In particular, Tree-based network optimizer (TORO) [6], which applies incremental pose parametrization and a tree structure on top of SGD, has been reported to be extremely robust to poor initial values, especially when the covariance matrix of the relative pose is close to spherical.

These phenomena show that the nonlinear optimization problem involved in pose graph SLAM appears to have a very special underling structure that requires further investigation. Since loop closing is an important feature in SLAM optimization problems, we start from analyzing the "minimal pose graph SLAM problem with loop closure", namely, the pose graph with 3 poses and 3 constraints. Analyzing minimal problems (e.g. http://cmp.felk.cvut.cz/minimal/ and [7]) can provide us some insights into the fundamental structure and properties of the large problems.

In this paper, based on our initial work on the analysis of the number of minima for point feature based SLAM problems [8], we prove that solving the basic 3-pose 3-constraint pose graph as a least squares problem is equivalent to solving a one dimensional optimization problem. Furthermore, we extend the result to pose graph with "two anchor nodes" and show how to obtain the global minimum of the one dimensional optimization problem 1 .

The paper is organized as follows. Section II presents the least squares formulation of the 3-pose 3-constraint pose graph SLAM problem and shows its equivalence to a one dimensional optimization problem. In Section III, the result is extended to the pose graph with only two anchor nodes. Section IV presents some properties of the one dimensional optimization problem. Section $\mathrm{V}$ discusses the related work and potential applications of the results. Finally Section VI concludes the paper. The proofs of the two main results in this paper are given in the Appendix.

\section{Pose Graph with 3 Poses and 3 Constraints}

Suppose there are three $2 \mathrm{D}$ robot poses $r_{0}, r_{1}, r_{2}$ and three constraints $Z_{1}^{0}, Z_{2}^{0}, Z_{2}^{1}$ where $Z_{j}^{i}$ is the relative pose constraint

\footnotetext{
${ }^{1}$ The MATLAB source code for solving this kind of pose graph SLAM

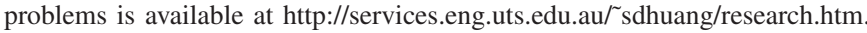




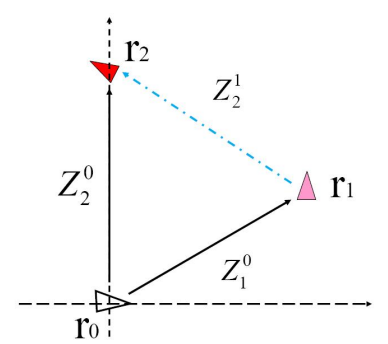

Fig. 1. Pose graph with 3 poses and 3 constraints.

from pose $r_{i}$ to pose $r_{j}(i=0,1, j=1,2)$. The corresponding pose graph is shown in Fig. 1 with 3 nodes (poses) and 3 edges (relative pose constraints).

\section{A. Least Squares Problem Formulation}

We choose pose $r_{0}$ as the origin of the global coordinate frame. That is, $r_{0}=\left(x_{r_{0}}, y_{r_{0}}, \phi_{r_{0}}\right)^{T}=(0,0,0)^{T}$. The least squares problem is to minimize

$$
F(X)=\sum_{i, j}\left(Z_{j}^{i}-H^{Z_{j}^{i}}(X)\right)^{T} P_{Z_{j}^{i}}^{-1}\left(Z_{j}^{i}-H^{Z_{j}^{i}}(X)\right)
$$

where $Z_{j}^{i}$ is the relative pose constraint from pose $r_{i}$ to pose $r_{j}$, and $P_{Z_{j}^{i}}$ is the corresponding covariance matrix. The function $H^{Z_{j}^{0}}(X)$ is given by

$$
H^{Z_{j}^{0}}(X)=\left[\begin{array}{lll}
x_{r_{j}} & y_{r_{j}} & \phi_{r_{j}}
\end{array}\right]^{T}
$$

and the function $H^{Z_{j}^{1}}(X)$ is given by 2

$$
H^{Z_{j}^{1}}(X)=\left[\begin{array}{c}
c_{\phi_{r_{1}}}\left(x_{r_{j}}-x_{r_{1}}\right)+s_{\phi_{r_{1}}}\left(y_{r_{j}}-y_{r_{1}}\right) \\
-s_{\phi_{r_{1}}}\left(x_{r_{j}}-x_{r_{1}}\right)+c_{\phi_{r_{1}}}\left(y_{r_{j}}-y_{r_{1}}\right) \\
\phi_{r_{j}}-\phi_{r_{1}}
\end{array}\right]
$$

For the 3-pose 3-constraint problem, the state vector $X$ is

$$
X=\left[\begin{array}{llllll}
x_{r_{2}} & y_{r_{2}} & \phi_{r_{2}} & x_{r_{1}} & y_{r_{1}} & \phi_{r_{1}}
\end{array}\right]^{T} .
$$

Suppose the relative pose constraints are

$$
\begin{aligned}
& Z_{1}^{0}=\left(z_{x_{r_{1}}}, z_{y_{r_{1}}}, z_{\phi_{r_{1}}}\right)^{T} \\
& Z_{2}^{0}=\left(z_{x_{r_{2}}}, z_{y_{r_{2}}}, z_{\phi_{r_{2}}}\right)^{T} \\
& Z_{2}^{1}=\left(z_{x_{r_{2}}^{r_{1}}}, z_{y_{r_{2}}}, z_{\phi_{r_{2}}}\right)^{T} .
\end{aligned}
$$

Assume the covariance matrices $P_{Z_{j}^{i}}$ are spherical 3

$$
\begin{aligned}
P_{Z_{1}^{0}} & =\operatorname{diag}\left(\gamma_{r_{1}}^{-1}, \gamma_{r_{1}}^{-1}, \gamma_{\phi_{r_{1}}}^{-1}\right) \\
P_{Z_{2}^{0}} & =\operatorname{diag}\left(\gamma_{r_{2}}^{-1}, \gamma_{r_{2}}^{-1}, \gamma_{\phi_{r_{2}}}^{-1}\right) \\
P_{Z_{2}^{1}} & =\operatorname{diag}\left(\gamma_{r_{2} r_{1}}^{-1}, \gamma_{r_{2} r_{1}}^{-1}, \gamma_{\phi_{r_{2}}}^{-1}\right)
\end{aligned}
$$

\footnotetext{
${ }^{2}$ In this paper, we use $c_{\phi_{r_{1}}}, s_{\phi_{r_{1}}}, c_{z_{\phi_{r_{1}}}}, s_{z_{\phi_{r_{1}}}}$ to denote $\cos \left(\phi_{r_{1}}\right)$, $\sin \left(\phi_{r_{1}}\right), \cos \left(z_{\phi_{r_{1}}}\right), \sin \left(z_{\phi_{r_{1}}}\right)$ respectively.

${ }^{3}$ Here spherical means diagonal with the first two elements (corresponding to $x$ and $y$ ) being the same. Most of the publicly available datasets (e.g. [9]) have the covariance matrices in this format.
}

then the objective function is

$$
\begin{aligned}
& F(X) \\
& =\gamma_{r_{1}}\left[\left(z_{x_{r_{1}}}-x_{r_{1}}\right)^{2}+\left(z_{y_{r_{1}}}-y_{r_{1}}\right)^{2}\right]+\gamma_{\phi_{r_{1}}}\left(z_{\phi_{r_{1}}}-\phi_{r_{1}}\right)^{2} \\
& +\gamma_{r_{2}}\left[\left(z_{x_{r_{2}}}-x_{r_{2}}\right)^{2}+\left(z_{y_{r_{2}}}-y_{r_{2}}\right)^{2}\right]+\gamma_{\phi_{r_{2}}}\left(z_{\phi_{r_{2}}}-\phi_{r_{2}}\right)^{2} \\
& +\gamma_{r_{2} r_{1}}\left[z_{x_{r_{2}}^{r_{1}}}-c_{\phi_{r_{1}}}\left(x_{r_{2}}-x_{r_{1}}\right)-s_{\phi_{r_{1}}}\left(y_{r_{2}}-y_{r_{1}}\right)\right]^{2} \\
& +\gamma_{r_{2} r_{1}}\left[z_{y_{r_{2}}^{r_{1}}}+s_{\phi_{r_{1}}}\left(x_{r_{2}}-x_{r_{1}}\right)-c_{\phi_{r_{1}}}\left(y_{r_{2}}-y_{r_{1}}\right)\right]^{2} \\
& +\gamma_{\phi_{r_{2}} r_{1}}\left(z_{\phi_{r_{2}}^{r_{1}}}-\phi_{r_{2}}+\phi_{r_{1}}\right)^{2} .
\end{aligned}
$$

Note that $F(X)$ can also be written in a new form

$$
\begin{aligned}
& F(X) \\
& =\gamma_{r_{1}}\left[\left(z_{x_{r_{1}}}-x_{r_{1}}\right)^{2}+\left(z_{y_{r_{1}}}-y_{r_{1}}\right)^{2}\right]+\gamma_{\phi_{r_{1}}}\left(z_{\phi_{r_{1}}}-\phi_{r_{1}}\right)^{2} \\
& +\gamma_{r_{2}}\left[\left(z_{x_{r_{2}}}-x_{r_{2}}\right)^{2}+\left(z_{y_{r_{2}}}-y_{r_{2}}\right)^{2}\right]+\gamma_{\phi_{r_{2}}}\left(z_{\phi_{r_{2}}}-\phi_{r_{2}}\right)^{2} \\
& +\gamma_{r_{2} r_{1}}\left[A\left(\phi_{r_{1}}\right)-\left(x_{r_{2}}-x_{r_{1}}\right)\right]^{2} \\
& +\gamma_{r_{2} r_{1}}\left[B\left(\phi_{r_{1}}\right)-\left(y_{r_{2}}-y_{r_{1}}\right)\right]^{2} \\
& +\gamma_{\phi_{r_{2}}^{r_{1}}}\left(z_{\phi_{r_{2}}^{r_{1}}}-\phi_{r_{2}}+\phi_{r_{1}}\right)^{2}
\end{aligned}
$$

where 4

$$
\begin{aligned}
& A\left(\phi_{r_{1}}\right)=z_{x_{r_{2}}^{r_{1}}} c_{\phi_{r_{1}}}-z_{y_{r_{2}}^{r_{1}}} s_{\phi_{r_{1}}}, \\
& B\left(\phi_{r_{1}}\right)=z_{x_{r_{2}}^{r_{1}}} s_{\phi_{r_{1}}}+z_{y_{r_{2}}^{r_{1}}} c_{\phi_{r_{1}}}
\end{aligned}
$$

Remark 1. In (7), the term $c_{\phi_{r_{1}}}$ and $s_{\phi_{r_{1}}}$ are separated from the term $\left(x_{r_{2}}-x_{r_{1}}\right)$ and $\left(y_{r_{2}}-y_{r_{1}}\right)$ which make the computation of the gradient and analyzing of the stationary points easier. It is clear from our results that spherical covariance in the form (5) can simplify the problem significantly.

\section{B. The Equivalence to One Dimensional Problem}

The following theorem shows that the problem of minimizing the objective function (7) is equivalent to an optimization problem with one variable.

The results in this paper hold for general spherical covariance matrices as given in (5). However, in order to simplify the formulas and make the paper more readable, in the reminder of this paper, we assume

$$
\gamma_{r_{1}}=\gamma_{\phi_{r_{1}}}=\gamma_{r_{2}}=\gamma_{\phi_{r_{2}}}=\gamma_{r_{2} r_{1}}=\gamma_{\phi_{r_{2}}^{r_{1}}}=1 \text {. }
$$

Theorem 1: Given data $z_{x_{r_{1}}}, z_{y_{r_{1}}}, z_{\phi_{r_{1}}}, z_{x_{r_{2}}}, z_{y_{r_{2}}}, z_{\phi_{r_{2}}}$, and $z_{x_{r_{2}}^{r_{1}}}, z_{y_{r_{2}}^{r_{1}}}, z_{\phi_{r_{2}}^{r_{1}}}$, we have that minimizing the objective function (7) under assumption (9) is equivalent to minimizing the following function of one variable $\phi$ :

$$
f(\phi)=\phi^{2}+\frac{1}{2}\left(\phi+\Delta z_{\phi_{r_{2}}^{r_{1}}}\right)^{2}-2 a \cos (\phi+\alpha)+b
$$

where $\Delta z_{\phi_{r_{2}}^{r_{1}}}, a, \alpha, b$ are constants that can be computed from the data by

$$
\begin{aligned}
& \Delta z_{\phi_{r_{2}}^{r_{1}}}=z_{\phi_{r_{2}}^{r_{1}}-z_{\phi_{r_{2}}}}+z_{\phi_{r_{1}}} \in[-\pi, \pi) \\
& a=\sqrt{p^{2}+(d+q)^{2}} \\
& \alpha=\operatorname{atan} 2(p, d+q) \\
& b=\frac{1}{3}\left[z_{x_{r_{2}}^{r_{1}}}^{2}+z_{y_{r_{2}}^{r_{1}}}^{2}+\left(z_{x_{r_{2}}}-z_{x_{r_{1}}}\right)^{2}+\left(z_{y_{r_{2}}}-z_{y_{r_{1}}}\right)^{2}\right]
\end{aligned}
$$

${ }^{4}$ In the following, we will simply use $A, B$ to denote the functions $A\left(\phi_{r_{1}}\right), B\left(\phi_{r_{1}}\right)$. This also applies to $A_{i}, B_{i}, A_{2 k}, B_{2 k}$ etc. 
TABLE I

EXAMPLE 1: FOUR DATA WITH DifFERENT LEVEL of NOISES. THE GROUND TRUTH OF THE POSES ARE $r_{0}=(0,0,0), r_{1}=\left(1,0.5, \frac{\pi}{2}\right), r_{2}=\left(0,1, \frac{3}{4} \pi\right)$.

\begin{tabular}{|c|c|c|c|c|c|c|c|c|c|}
\hline noise & $\left(z_{x_{r_{1}}}, z_{y_{r_{1}}}, z_{\phi_{r_{1}}}\right)$ & $\left(z_{x_{r_{2}}}, z_{y_{r_{2}}}, z_{\phi_{r_{2}}}\right)$ & $\left(z_{x_{r_{2}}^{r_{1}}}, z_{y_{r_{2}}^{r_{1}}}, z_{\phi_{r_{2}}^{r_{1}}}\right)$ & $\Delta z_{\phi_{r_{2}}^{r_{1}}}$ & $\alpha$ & No. & $\phi^{*}$ & $f\left(\phi^{*}\right)$ & $F\left(X^{*}\right)$ \\
\hline zero & $(1.0000,0.5000,1.5708)$ & $(0,1.0000,2.3562)$ & $(0.5000,1.0000,0.7854)$ & 0 & 0 & 1 & 0 & 0 & 0 \\
\hline small & $(1.10220 .39671 .5383)$ & $\left(\begin{array}{llll}0.0805 & 0.9991 & 2.5293\end{array}\right)$ & $\left(\begin{array}{llll}0.6335 & 0.9682 & 0.8705\end{array}\right)$ & -0.1204 & -0.0793 & 1 & 0.0493 & 0.0057 & 0.0057 \\
\hline large & $\left(\begin{array}{llll}0.9368 & 0.6861 & 2.1437\end{array}\right)$ & $\left(\begin{array}{llll}-0.2468 & 1.0940 & 2.3901)\end{array}\right.$ & $\left(\begin{array}{llll}0.5627 & 1.3630 & 1.1482\end{array}\right)$ & 0.9018 & 0.5132 & 1 & -0.3623 & 0.3073 & 0.3073 \\
\hline huge & $(1.16746 .13750 .0755)$ & $\left(\begin{array}{llll}-6.6684 & 2.7509 & 2.4706\end{array}\right)$ & $(-0.8100-7.7511-0.6429)$ & -3.0380 & 1.1342 & 3 & -0.9978 & 9.7355 & 9.7355 \\
\hline
\end{tabular}

'No.' means "the number of minima in $[-2 \pi-\alpha, 2 \pi-\alpha]$ ".

where

$$
\begin{aligned}
& d=\frac{1}{3}\left[\left(z_{x_{r_{2}}}-z_{x_{r_{1}}}\right)^{2}+\left(z_{y_{r_{2}}}-z_{y_{r_{1}}}\right)^{2}\right] \\
& p=\delta_{a} c_{z_{\phi_{1}}}+\delta_{b} s_{z_{\phi_{1}}} \\
& q=-\delta_{a} s_{z_{\phi_{r_{1}}}}+\delta_{b} c_{z_{\phi_{r_{1}}}} \\
& \delta_{a}=-\Delta z_{x_{r_{2}}^{r_{1}}}\left(-\frac{1}{3} z_{y_{r_{1}}}+\frac{1}{3} z_{y_{r_{2}}}\right)+\Delta z_{y_{r_{2}}^{r_{1}}}\left(-\frac{1}{3} z_{x_{r_{1}}}+\frac{1}{3} z_{x_{r_{2}}}\right) \\
& \delta_{b}=\Delta z_{x_{r_{2}}^{r_{1}}}\left(-\frac{1}{3} z_{x_{r_{1}}}+\frac{1}{3} z_{x_{r_{2}}}\right)+\Delta z_{y_{r_{2}}^{r_{1}}}\left(-\frac{1}{3} z_{y_{r_{1}}}+\frac{1}{3} z_{y_{r_{2}}}\right) \\
& \Delta z_{x_{r_{2}}^{r_{1}}}=z_{x_{r_{2}}^{r_{1}}}-\left(z_{x_{r_{2}}}-z_{x_{r_{1}}}\right) c_{z_{\phi_{r_{1}}}}-\left(z_{y_{r_{2}}}-z_{y_{r_{1}}}\right) s_{z_{\phi_{r_{1}}}} \\
& \Delta z_{y_{r_{2}}^{r_{1}}}=z_{y_{r_{2}}^{r_{1}}}+\left(z_{x_{r_{2}}}-z_{x_{r_{1}}}\right) s_{z_{\phi_{r_{1}}}}-\left(z_{y_{r_{2}}}-z_{y_{r_{1}}}\right) c_{z_{\phi_{r_{1}}}} \text {. }
\end{aligned}
$$

In fact, once the solution $\phi$ to the one variable optimization problem is obtained, the solution to the problem of minimizing $F(X)$ in (7) can be obtained in a closed-form by the following formulas

$$
\begin{aligned}
\phi_{r_{1}} & =\phi+z_{\phi_{r_{1}}} \\
\phi_{r_{2}} & =\frac{1}{2}\left(z_{\phi_{r_{2}}}+z_{\phi_{r_{2}}}+\phi_{r_{1}}\right) \\
x_{r_{1}} & =\frac{2}{3} z_{x_{r_{1}}}+\frac{1}{3} z_{x_{r_{2}}}-\frac{1}{3} A \\
y_{r_{1}} & =\frac{2}{3} z_{y_{r_{1}}}+\frac{1}{3} z_{y_{r_{2}}}-\frac{1}{3} B \\
x_{r_{2}} & =\frac{1}{3} z_{x_{r_{1}}}+\frac{2}{3} z_{x_{r_{2}}}+\frac{1}{3} A \\
y_{r_{2}} & =\frac{1}{3} z_{y_{r_{1}}}+\frac{2}{3} z_{y_{r_{2}}}+\frac{1}{3} B .
\end{aligned}
$$

Proof: See Appendix A

Remark 2. The constants $\Delta z_{\phi_{r_{2}}}, a, \alpha, b$ in $f(\phi)$ summarize some important information from the relative pose constraints data. For example, $\Delta z_{\phi_{r_{2}}^{r_{1}}}$ indicates the level of consistency among the three angles $z_{\phi_{r_{2}}^{r_{1}}}, z_{\phi_{r_{2}}}$, and $z_{\phi_{r_{1}}}$. If the three angles are compatible with each other (e.g. when the relative orientation estimates are accurate), then $\Delta z_{\phi_{r_{2}}^{r_{1}}}$ is close to 0 . Similarly, if the relative pose data are accurate, then $\alpha$ is close to 0 and $a$ is proportional to the square of the distance between $r_{1}$ and $r_{2}$. Constant $b$ does not have any impact on the solution to the optimization problem.

Example 1. To illustrate the results in Theorem 1, consider the following example. Assume the ground truth of the poses are $r_{0}=(0,0,0), r_{1}=\left(1,0.5, \frac{\pi}{2}\right), r_{2}=\left(0,1, \frac{3}{4} \pi\right)$ which is similar to the configuration in Fig. 1. Four examples of relative constraints datasets randomly generated with 4 different levels of noise are listed in Table I. The corresponding constants $\Delta z_{\phi_{r_{2}}^{r_{1}}}, a, \alpha, b$ can then be computed for each dataset using the formulas in (11) and the function $f(\phi)$ in (10) can be obtained. The two key constants $\Delta z_{\phi_{r_{2}}^{r_{1}}}$ and $\alpha$, the number of local minima within $[-2 \pi-\alpha, 2 \pi-\alpha]$, the global minimum $\phi^{*}$ and $f\left(\phi^{*}\right)$, as well as the objective function value obtained through solving the least squares problem using Gauss-Newton $\left(F\left(X^{*}\right)\right)$ are all listed in Table I. Fig. 2 shows the function $f(\phi)$ for the 4 different datasets. It can be seen that more than one minima exist only when the noise is unrealistically large.

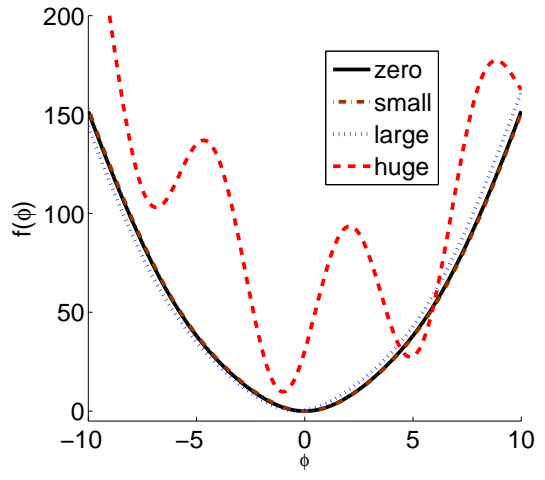

Fig. 2. The function $f(\phi)$ in Theorem 1 for datasets with different levels of noise: pose graph with 3 poses and 3 constraints.

\section{Pose Graph with Two Anchor Poses}

Now consider a more general pose graph as shown in Fig. 3 This pose graph has a special structure. Namely, all the edges are connecting one of the two nodes $r_{0}$ and $r_{1}$. Here we call nodes $r_{0}$ and $r_{1}$ "anchor nodes" or "anchor poses".

Assume there are:

- $n$ poses that have two edges linked to both $r_{0}$ and $r_{1}$ : $r_{2}, \cdots, r_{n}, r_{n+1}$

- $n_{1}$ poses that only have one edge linked to $r_{0}$ : $r_{11}, r_{12}, \cdots, r_{1 n_{1}}$

- $n_{2}$ poses that only have one edge linked to $r_{1}$ : $r_{21}, r_{22}, \cdots, r_{2 n_{2}}$.

Assume the relative pose constraints are:

- from $r_{0}$ to $r_{1}:\left(z_{x_{r_{1}}}, z_{y_{r_{1}}}, z_{\phi_{r_{1}}}\right)^{T}$

- from $r_{0}$ to $r_{i}:\left(z_{x_{r_{i}}}, z_{y_{r_{i}}}, z_{\phi_{r_{i}}}\right)^{T}, i=2, \ldots, n+1$;

- from $r_{1}$ to $r_{i}:\left(z_{x_{i}}^{r_{1}}, z_{y_{r_{1}}}^{r_{1}}, z_{\phi_{r_{i}}^{r_{1}}}\right)^{T}, i=2, \ldots, n+1$;

- from $r_{0}$ to $r_{1 j}:\left(z_{x_{r_{1 j}}}, z_{y_{r_{1 j}}}, z_{\phi_{r_{1 j}}}\right)^{T}, j=1, \ldots, n_{1}$;

- from $r_{1}$ to $r_{2 k}:\left(z_{x_{r_{2 k}}}, z_{y_{r_{2 k}}}, z_{\phi_{r_{2 k}}}\right)^{T}, k=1, \ldots, n_{2}$.

In the example shown in Fig. 3, $n=3, n_{1}=2, n_{2}=4$. It is obvious that the 3-pose 3-constraint pose graph in Fig. 1 is a special case with $n=1, n_{1}=0, n_{2}=0$.

The covariance matrices of the relative pose constraints can be assumed to have the same format as in (5). However, to simplify the formulas, we assume the covariance matrices are all identity matrices.

To find the optimal configuration of the pose graph, the state vector $X$ contains all the robot poses except pose $r_{0}$ which is the origin of the coordinate frame. Similar to (7), the objective function of the least squares optimization problem 


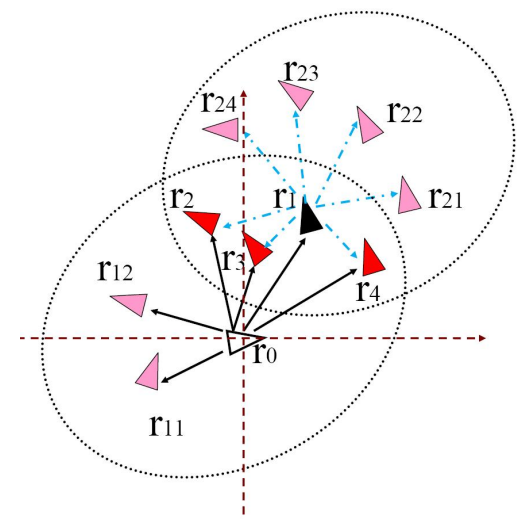

Fig. 3. Pose graph with two anchor poses $r_{0}$ and $r_{1}$.

can be written as

$$
\begin{aligned}
& F(X)=\left(z_{x_{r_{1}}}-x_{r_{1}}\right)^{2}+\left(z_{y_{r_{1}}}-y_{r_{1}}\right)^{2}+\left(z_{\phi_{r_{1}}}-\phi_{r_{1}}\right)^{2} \\
& +\sum_{i=2}^{n+1}\left[\left(z_{x_{r_{i}}}-x_{r_{i}}\right)^{2}+\left(z_{y_{r_{i}}}-y_{r_{i}}\right)^{2}+\left(z_{\phi_{r_{i}}}-\phi_{r_{i}}\right)^{2}\right. \\
& +\left(A_{i}-\left(x_{r_{i}}-x_{r_{1}}\right)\right)^{2}+\left(B_{i}-\left(y_{r_{i}}-y_{r_{1}}\right)\right)^{2} \\
& \left.+\left(z_{\phi_{r_{i}}^{r_{1}}}-\left(\phi_{r_{i}}-\phi_{r_{1}}\right)\right)^{2}\right] \\
& +\sum_{j=1}^{n_{1}}\left[\left(z_{x_{r_{1 j}}}-x_{r_{1 j}}\right)^{2}+\left(z_{y_{r_{1 j}}}-y_{r_{1 j}}\right)^{2}\right. \\
& \left.+\left(z_{\phi_{r_{1 j}}}-\phi_{r_{1 j}}\right)^{2}\right]+\sum_{k=1}^{n_{2}}\left[\left(A_{2 k}-\left(x_{r_{2 k}}-x_{r_{1}}\right)\right)^{2}\right. \\
& \left.+\left(B_{2 k}-\left(y_{r_{2 k}}-y_{r_{1}}\right)\right)^{2}+\left(z_{\phi_{r_{2 k}}^{r_{1}}}-\left(\phi_{r_{2 k}}-\phi_{r_{1}}\right)\right)^{2}\right]
\end{aligned}
$$

where for $i=2, \ldots, n+1, k=1, \ldots, n_{2}$,

$$
\begin{aligned}
& A_{i}=z_{x_{r_{i}}^{r_{1}}} c_{\phi_{r_{1}}}-z_{y_{r_{i}}^{r_{1}}} s_{\phi_{r_{1}}}, B_{i}=z_{x_{r_{i}}^{r_{1}}} s_{\phi_{r_{1}}}+z_{y_{r_{i}}^{r_{1}}} c_{\phi_{r_{1}}}, \\
& A_{2 k}=z_{x_{r_{2 k}}^{r_{1}}} c_{\phi_{r_{1}}}-z_{y_{r_{2 k}}^{r_{1}}} s_{\phi_{r_{1}}}, B_{2 k}=z_{x_{r_{2 k}}^{r_{1}}} s_{\phi_{r_{1}}}+z_{y_{r_{2 k}}^{r_{1}}} c_{\phi_{r_{1}}} \text {. }
\end{aligned}
$$

The following theorem demonstrates that minimizing (14) is equivalent to a one dimensional optimization problem.

Theorem 2: The problem of minimizing the objective function (14) is equivalent to minimizing the following function with one variable $\phi$ :

$$
f(\phi)=\phi^{2}+\frac{1}{2} \sum_{i=2}^{n+1}\left[\phi+\Delta z_{\phi_{r_{i}}^{r_{1}}}\right]^{2}-2 a \cos (\phi+\alpha)+b
$$

where $\Delta z_{\phi_{r_{j}}^{r_{1}}}, a, \alpha$ and $b$ can be computed from the relative constraints data by

$$
\begin{aligned}
\Delta z_{\phi_{r_{i}}^{r_{1}}} & =z_{\phi_{r_{i}}^{r_{1}}}-\left(z_{\phi_{r_{i}}}-z_{\phi_{r_{1}}}\right) \in[-\pi, \pi], \quad i=2, \cdots, n+1, \\
a & =\sqrt{p^{2}+(d+q)^{2}}, \\
\alpha & =\operatorname{atan} 2(p, d+q),
\end{aligned}
$$

$$
\begin{aligned}
b= & \left(\frac{1}{n+2} \sum_{i=2}^{n+1} z_{x_{r_{i}}}+\left(\frac{2}{n+2}-1\right) z_{x_{r_{1}}}\right)^{2} \\
& +\left(\frac{1}{n+2} \sum_{i=2}^{n+1} z_{y_{r_{i}}}+\left(\frac{2}{n+2}-1\right) z_{y_{r_{1}}}\right)^{2} \\
& +2 \sum_{j=2}^{n+1}\left[\frac{1}{n+2} z_{x_{r_{1}}}-\frac{1}{2} z_{x_{r_{j}}}+\frac{1}{2(n+2)} \sum_{i=2}^{n+1} z_{x_{r_{i}}}\right]^{2} \\
& +2 \sum_{j=2}^{n+1}\left[\frac{1}{n+2} z_{y_{r_{1}}}-\frac{1}{2} z_{y_{r_{j}}}+\frac{1}{2(n+2)} \sum_{i=2}^{n+1} z_{y_{r_{i}}}\right]^{2} \\
& +\frac{n+1}{2(n+2)} \sum_{i=2}^{n+1}\left(z_{x_{r_{i}}^{r_{1}}}^{2}+z_{y_{r_{i}}^{r_{1}}}^{2}\right) \\
& -\frac{1}{n+2} \sum_{2 \leq i<j \leq n+1}\left(z_{x_{r_{i}}^{r_{1}} z_{x_{r_{j}}}^{r_{1}}}+z_{y_{r_{i}}^{r_{1}}} z_{y_{r_{j}}^{r_{1}}}\right)
\end{aligned}
$$

where

$$
\begin{aligned}
& d=\frac{1}{2} \sum_{2 \leq i \leq n+1}\left[\left(z_{x_{r_{i}}}-z_{x_{r_{1}}}\right)^{2}+\left(z_{y_{r_{i}}}-z_{y_{r_{1}}}\right)^{2}\right] \\
& -\frac{1}{2(n+2)} \sum_{2 \leq i, j \leq n+1}\left[\left(z_{x_{r_{j}}}-z_{x_{r_{1}}}\right)\left(z_{x_{r_{i}}}-z_{x_{r_{1}}}\right)\right. \\
& \left.+\left(z_{y_{r_{j}}}-z_{y_{r_{1}}}\right)\left(z_{y_{r_{i}}}-z_{y_{r_{1}}}\right)\right] \\
& p=\delta_{a} c_{z_{\phi_{r_{1}}}}+\delta_{b} s_{z_{\phi_{r_{1}}}} \\
& q=-\delta_{a} s_{z_{\phi_{r_{1}}}}+\delta_{b} c_{z_{\phi_{r_{1}}}}, \\
& \delta_{a}=\sum_{j=2}^{n+1}\left(\left[\begin{array}{l}
\Delta z_{x_{r_{j}}}^{r_{1}} \\
\Delta z_{y_{r_{j}}}^{r_{1}}
\end{array}\right]^{T}\left[\begin{array}{l}
\frac{1}{n+2} z_{y_{r_{1}}}-\frac{1}{2} z_{y_{r_{j}}}+\frac{1}{2(n+2)} \sum_{i=2}^{n+1} z_{y_{r_{i}}} \\
\frac{-1}{n+2} z_{x_{r_{1}}}+\frac{1}{2} z_{x_{r_{j}}}-\frac{1}{2(n+2)} \sum_{i=2}^{n+1} z_{x_{r_{i}}}
\end{array}\right]\right), \\
& \delta_{b}=\sum_{j=2}^{n+1}\left(\left[\begin{array}{l}
\Delta z_{x_{r_{j}}^{r_{1}}} \\
\Delta z_{y_{r_{j}}^{r_{1}}}
\end{array}\right]^{T}\left[\begin{array}{l}
\frac{-1}{n+2} z_{x_{r_{1}}}+\frac{1}{2} z_{x_{r_{j}}}-\frac{1}{2(n+2)} \sum_{i=2}^{n+1} z_{x_{r_{i}}} \\
\frac{-1}{n+2} z_{y_{r_{1}}}+\frac{1}{2} z_{y_{r_{j}}}-\frac{1}{2(n+2)} \sum_{i=2}^{n+1} z_{y_{r_{i}}}
\end{array}\right]\right), \\
& \Delta z_{x_{r_{i}}^{r_{1}}}=z_{x_{r_{i}}^{r_{1}}}-\left[\left(z_{x_{r_{i}}}-z_{x_{r_{1}}}\right) c_{z_{\phi_{r_{1}}}}+\left(z_{y_{r_{i}}}-z_{y_{r_{1}}}\right) s_{z_{\phi_{r_{1}}}}\right] \text {, } \\
& \Delta z_{y_{r_{i}}^{r_{1}}}=z_{y_{r_{i}}^{r_{1}}}-\left[-\left(z_{x_{r_{i}}}-z_{x_{r_{1}}}\right) s_{z_{\phi_{r_{1}}}}+\left(z_{y_{r_{i}}}-z_{y_{r_{1}}}\right) c_{z_{\phi_{r_{1}}}}\right] \text {, } \\
& i=2, \cdots, n+1 \text {. }
\end{aligned}
$$

In fact, once the solution $\phi$ to the one variable optimization problem is obtained, the $X$ that minimizes (14) can be obtained in a closed-form by the following formulas:

(i) For pose $r_{1 j},\left(1 \leq j \leq n_{1}\right)$

$$
\phi_{r_{1 j}}=z_{\phi_{r_{1 j}}}, \quad x_{r_{1 j}}=z_{x_{r_{1 j}}}, \quad y_{r_{1 j}}=z_{y_{r_{1 j}}} .
$$

(ii) For pose $r_{1}$,

$$
\begin{aligned}
& \phi_{r_{1}}=\phi+z_{\phi_{r_{1}}} \\
& x_{r_{1}}=\frac{1}{n+2} \sum_{i=2}^{n+1}\left(z_{x_{r_{i}}}+A_{i}\right)+\frac{2}{n+2}\left(z_{x_{r_{1}}}-\sum_{i=2}^{n+1} A_{i}\right) \\
& y_{r_{1}}=\frac{1}{n+2} \sum_{i=2}^{n+1}\left(z_{y_{r_{i}}}+B_{i}\right)+\frac{2}{n+2}\left(z_{y_{r_{1}}}-\sum_{i=2}^{n+1} B_{i}\right)
\end{aligned}
$$


(iii) For pose $r_{i},(2 \leq i \leq n+1)$,

$$
\begin{aligned}
\phi_{r_{i}} & =\frac{1}{2}\left(z_{\phi_{r_{i}}}+z_{\phi_{r_{i}}^{r_{1}}}+\phi_{r_{1}}\right) \\
x_{r_{i}} & =\frac{1}{2}\left(z_{x_{r_{i}}}+A_{i}\right)+\frac{1}{2(n+2)} \sum_{j=2}^{n+1}\left(z_{x_{r_{j}}}+A_{j}\right) \\
& +\frac{1}{n+2}\left(z_{x_{r_{1}}}-\sum_{j=2}^{n+1} A_{j}\right) \\
y_{r_{i}} & =\frac{1}{2}\left(z_{y_{r_{i}}}+B_{i}\right)+\frac{1}{2(n+2)} \sum_{j=2}^{n+1}\left(z_{y_{r_{j}}}+B_{j}\right) \\
& +\frac{1}{n+2}\left(z_{y_{r_{1}}}-\sum_{j=2}^{n+1} B_{j}\right)
\end{aligned}
$$

(iv) For pose $r_{2 k},\left(1 \leq k \leq n_{2}\right)$

$$
\phi_{r_{2 k}}=z_{\phi_{r_{2 k}}^{r_{1}}}+\phi_{r_{1}}, x_{r_{2 k}}=x_{r_{1}}+A_{2 k}, y_{r_{2 k}}=y_{r_{1}}+B_{2 k} \text {. }
$$

Proof: The proof is similar to that of Theorem 1 and is omitted. One of the technical challenges involved is the computing of the inverse of a $(2 n+2) \times(2 n+2)$ matrix (see the proof of Theorem 1 in [8]) in order to solve the linear equations to get the closed-form formulas in (19) and (20).

Remark 3. The objective functions considered in (7) and (14) treat the orientation variables as real numbers instead of angles. Since the error in angles is limited to $[-\pi, \pi)$, a regularization term might be needed in some situations. The problem of obtaining the correct regularization term is discussed in details in [10]. Note that for the problem considered in Theorem 2, because there are only two anchor poses and each relative angle is within $[-\pi, \pi)$, the regularization term $2 k \pi$ with $k= \pm 1$ will be enough if needed. Once the regularization term is determined, minimizing the new objective function is still a one dimensional problem.

\section{Properties of the One Dimensional Problem}

Now we consider the properties of the one dimensional function in Theorem 2 (Theorem 1 is a special case of Theorem 2 with $\left.n=1, n_{1}=0, n_{2}=0\right)$.

Theorem 3: Assume that $a>0, \alpha \in[-\pi, \pi)$ are constants, $n \geq 1$ is a positive integer and $\Delta z_{\phi_{r_{i}}^{r_{1}}} \in[-\pi, \pi), i=$ $2, \cdots, n+1$ are all constants. Then we have that

(i) The optimal solution $\phi^{*}$ of minimizing function $f(\phi)$ in (15) satisfies $\phi^{*} \in[-2 \pi-\alpha, 2 \pi-\alpha]$.

(ii) If $a<1+\frac{n}{2}$, then $f(\phi)$ in (15) has one and only one minimum which is the global minimum.

(iii) If $a \geq 1+\frac{n}{2}$, then $f(\phi)$ in (15) has at least one and at most three local minima in $[-2 \pi-\alpha, 2 \pi-\alpha]$. And the exact number of local minima depends on the values of $f^{\prime}\left(\phi_{1}\right), f^{\prime}\left(\phi_{2}\right), f^{\prime}\left(\phi_{3}\right), f^{\prime}\left(\phi_{4}\right)$, where 5

$$
\begin{aligned}
& \phi_{1}=-2 \pi+\arccos \left(-\frac{n+2}{2 a}\right)-\alpha, \\
& \phi_{2}=-\arccos \left(-\frac{n+2}{2 a}\right)-\alpha, \\
& \phi_{3}=\arccos \left(-\frac{n+2}{2 a}\right)-\alpha, \\
& \phi_{4}=2 \pi-\arccos \left(-\frac{n+2}{2 a}\right)-\alpha .
\end{aligned}
$$

\footnotetext{
${ }^{5}$ The $\phi_{1}, \phi_{2}, \phi_{3}, \phi_{4}$ in the one dimension problem considered in Example 2 are shown in Fig. 4(d)
}

TABLE II

THE NUMBER OF LOCAL MINIMA FOR $f(\phi)$ IN (15) WHEN $a \geq 1+\frac{n}{2}$.

\begin{tabular}{|c|c|c|c|c|c|c|c|c|}
\hline$f^{\prime}(-2 \pi-\alpha)$ & $<0$ & $<0$ & $<0$ & $<0$ & $<0$ & $<0$ & $<0$ & $<0$ \\
\hline$f^{\prime}\left(\phi_{1}\right)$ & $>0$ & $>0$ & $>0$ & $>0$ & $>0$ & $\leq 0$ & $\leq 0$ & $\leq 0$ \\
\hline$f^{\prime}\left(\phi_{2}\right)$ & $<0$ & $<0$ & $<0$ & $\geq 0$ & $\geq 0$ & $<0$ & $<0$ & $<0$ \\
\hline$f^{\prime}\left(\phi_{3}\right)$ & $>0$ & $>0$ & $\leq 0$ & $>0$ & $>0$ & $>0$ & $>0$ & $\leq 0$ \\
\hline$f^{\prime}\left(\phi_{4}\right)$ & $<0$ & $\geq 0$ & $<0$ & $<0$ & $\geq 0$ & $<0$ & $\geq 0$ & $<0$ \\
\hline$f^{\prime}(2 \pi-\alpha)$ & $>0$ & $>0$ & $>0$ & $>0$ & $>0$ & $>0$ & $>0$ & $>0$ \\
\hline No. & 3 & 2 & 2 & 2 & 1 & 2 & 1 & 1 \\
\hline
\end{tabular}

' $1,2,3$ ' denote the number of minima in $[-2 \pi-\alpha, 2 \pi-\alpha]$.

and $f^{\prime}(\phi)$ is the first derivative of $f(\phi)$ given by

$$
f^{\prime}(\phi)=2 a \sin (\phi+\alpha)+(n+2) \phi+\sum_{i=2}^{n+1} \Delta z_{\phi_{r_{i}}^{r_{1}}} .
$$

All the 8 different cases are listed in Table II.

Proof: See Appendix B.

Remark 4. Theorem 3 shows that the global minimum of the one dimensional problem is in $[-2 \pi-\alpha, 2 \pi-\alpha]$ and there are at most three minima in this interval. So the one dimensional problem can be solved easily using a bisection algorithm.

Example 2. We use the Freiburg Indoor Building 079 dataset [9] to construct an example. There are 989 poses (nodes) and 1314 constraints (edges) in this dataset. The data is separated into two parts to construct two pose graphs. Each pose graph is optimized by performing a Gauss-Newton least squares optimization. The optimized configuration of the two pose graphs and the edges involved are shown in Fig. 4(a) and Fig. 4(b), respectively.

For each pose graph, the resulting optimized pose configuration is the relative pose estimate with respect to the origin of the pose graph. We simply ignore the correlation among the pose estimates and treat the optimized configuration as independent relative pose constraints from the origin to the other poses in the graph. We also assume the covariance matrices of the relative pose constraints are all identity.

Now we have got a pose graph with two anchor nodes, one anchor is the origin of the first pose graph $\left(r_{0}\right)$ and the other anchor is the origin of the second pose graph $\left(r_{1}\right)$. There are $n=39$ nodes which are linked with both $r_{0}$ and $r_{1}$ as shown in Fig. 4(c) Applying Theorem 2, we can get function $f(\phi)$ as shown in Fig. 4(d). This function has three local minima in $[-2 \pi-\alpha, 2 \pi-\alpha]$ and the global minimum is $\phi^{*}=0.0078$. The objective function value $f\left(\phi^{*}\right)=0.06009$ is the same as that obtained by solving the constructed pose graph as a $988 \times 3$ dimensional least squares problem. Fig. 4(c) shows the result of computing all the 988 nodes using $\phi^{*}$ by the closed-form formulas in Theorem 2.

\section{RELATED WORK AND Discussions}

The pose graph optimization problem considered in Theorem 2 has some similarity with the problem of estimating the relative transformation between two coordinate frames given two corresponding point sets [11]. The major differences are that our problem includes a relative pose information between 


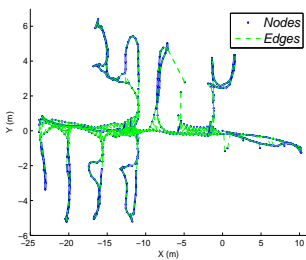

(a) The first pose graph.

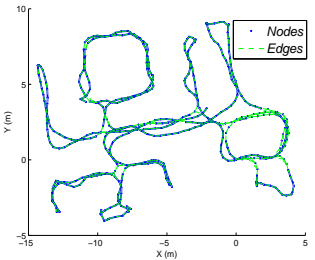

(b) The second pose graph.

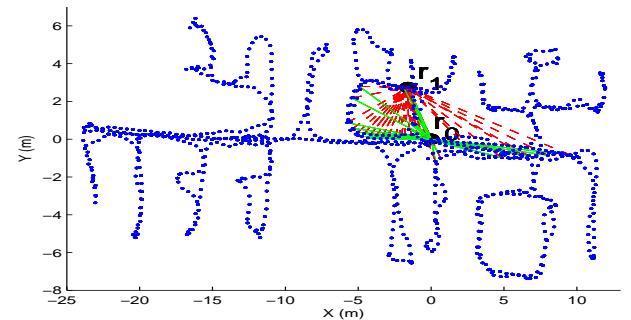

(c) The optimized pose graph with two anchor nodes (Only edges connecting the 39 common nodes are shown: solid edges linked to $r_{0}$ and dashed edges linked to $r_{1}$ ).

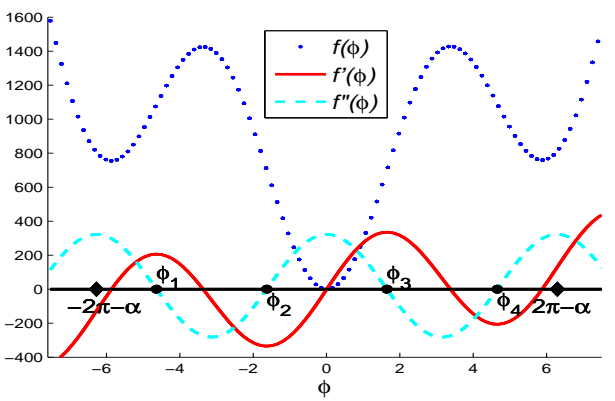

(d) The objective function of the one dimensional problem and its derivatives.

Fig. 4. Example of Theorem 2 using the Freiburg Indoor Building 079 dataset: pose graph with two anchor poses.

$r_{0}$ and $r_{1}$, and we are optimizing all the variables not only the relative pose.

Recently, an approximate solution to pose graph SLAM problem using linear estimation methods is proposed in [12] and then in [10]. The first step is to obtain a suboptimal estimate of all the robot orientations using linear method, then estimate all the variables using linear method. In our current work, we are proposing exact solutions instead of approximate solutions, and we just need to solve a one dimensional nonlinear problem to obtain one variable and all the other variables can then be calculated using closed-form formulas. However, our current results only hold for specific pose graphs.

The closest related work is [13] where it was attempted to compute a closed-form solution for pose-graph SLAM with identity covariance matrices by using $c_{\phi}=\cos (\phi)$ and $s_{\phi}=$ $\sin (\phi)$ as variables with an additional constraint $c_{\phi}^{2}+s_{\phi}^{2}=1$. However, only some initial results on computing the robot positions were derived in [13].
While the results in this paper provide some interesting properties of pose graph SLAM problems, they raise a number of questions in SLAM which require further investigation.

In pose graph SLAM, the property that the Jacobian and information matrix are exactly sparse [14] has been exploited in most of the recent efficient pose graph SLAM algorithms. Can we further exploit the special structure of the nonlinearity in pose graph SLAM such that more efficient algorithms can be developed? Or, has this structure already been implicitly used in the existing algorithms?

For example, SGD is proposed in [3]|6] to be used to solve the pose graph SLAM problems. This approach solves the optimization problem by dealing with each constraint oneby-one and can converge to good solutions most of the time even when the initial value is very poor. There might be some relationship between the 3-pose 3-constraint problem in our paper and the problem of dealing with one constraint in SGD. Moreover, since our results can be extended to pose graph with multiple constraints but only two anchor poses. It might be possible to deal with a set of constraints at a time when using SGD to speed up the algorithm further.

In practice, covariance matrices obtained from odometry and scan matching can have more general forms, however, most of the publicly available datasets has the spherical covariance in the format of equation (5). Our results show that spherical covariances can simplify the problem significantly. It might be valuable to introduce some datasets with nonspherical covariance matrices as benchmark datasets for testing different algorithms in the SLAM community.

The pose graph with two anchor nodes shown in Fig. 3 has similar structure with the problem of combining two optimized pose graphs using the map joining idea [15][16] which can be applied to large-scale SLAM or multi-robot SLAM [17]. However, in each of the optimized pose graph, all the poses are fully correlated and they cannot be simply treated as independent relative pose constraints as what we did in Example 2 ${ }^{6}$. So the result in Theorem 2 cannot be directly applied to combining two pose graphs although it can serve as a quick way to obtain a good initial value.

\section{CONCLUSION AND FUtURE WORK}

This paper provides some insights into the structure of the nonlinearities in the pose graph SLAM optimization problem. In particular, it shows that under the spherical covariance matrices assumption, optimizing a pose graph with only two anchor nodes is equivalent to a one dimensional optimization problem which has at most three local minima. The globally optimal pose configuration can thus be obtained by simply computing all the minima using a bisection algorithm.

In the next step, we would like to analyze the property of the SGD algorithm proposed in [3][6] where the constraints are dealt with once at a time. We will also consider the extension of the results to deal with more general covariance matrices

\footnotetext{
${ }^{6}$ The "good looking" result in Example 2 is probably due to the high quality of the data and the high quality of the two optimized pose graphs.
} 
and more general pose graph SLAM. Extension of the work to $3 \mathrm{D}$ scenarios will also be a nontrivial contribution.

\section{ACKNOWLEDGMENTS}

This work has been supported in part by the Funds of National Science of China (Grant No. 61004061) and Australian Research Council Discovery project (DP120102786).

\section{APPENDIX}

\section{A. Proof of Theorem 1}

Since the objective function $F(X)$ is smooth, the optimal solution that minimizes $F(X)$ must be a stationary point with zero gradient, that is $\nabla F(X)=0$.

By the definition of $A, B$ in (8), we have

$$
\frac{d A}{d \phi_{r_{1}}}=-B, \frac{d B}{d \phi_{r_{1}}}=A .
$$

Thus for $F(X)$ in (7) with assumption (9), we have

$$
\begin{aligned}
& \nabla F(X) \\
& =\left[\begin{array}{llllll}
\frac{\partial F(X)}{\partial x_{r_{2}}} & \frac{\partial F(X)}{\partial y_{r_{2}}} & \frac{\partial F(X)}{\partial \phi_{r_{2}}} & \frac{\partial F(X)}{\partial x_{r_{1}}} & \frac{\partial F(X)}{\partial y_{r_{1}}} & \frac{\partial F(X)}{\partial \phi_{r_{1}}}
\end{array}\right]^{T} \\
& =2\left[\begin{array}{c}
-\left(z_{x_{r_{2}}}-x_{r_{2}}\right)-\left(A-x_{r_{2}}+x_{r_{1}}\right) \\
-\left(z_{y_{r_{2}}}-y_{r_{2}}\right)-\left(B-y_{r_{2}}+y_{r_{1}}\right) \\
-\left(z_{\phi_{r_{2}}}-\phi_{r_{2}}\right)-\left(z_{\phi_{r_{2}}}-\phi_{r_{2}}+\phi_{r_{1}}\right) \\
-\left(z_{x_{r_{1}}}-x_{r_{1}}\right)+\left(A-x_{r_{2}}+x_{r_{1}}\right) \\
-\left(z_{y_{r_{1}}}-y_{r_{1}}\right)+\left(B-y_{r_{2}}+y_{r_{1}}\right) \\
\Theta
\end{array}\right]
\end{aligned}
$$

where

$\Theta=2 \phi_{r_{1}}-z_{\phi_{r_{1}}}+z_{\phi_{r_{2}}^{r_{1}}}-\phi_{r_{2}}+B\left(x_{r_{2}}-x_{r_{1}}\right)-A\left(y_{r_{2}}-y_{r_{1}}\right)$.

For any stationary point $X$, we can simplify the function $F(X)$ using the first 5 equations of $\nabla F(X)=0$ to get

$$
\begin{aligned}
F(X)= & \left(z_{\phi_{r_{1}}}-\phi_{r_{1}}\right)^{2}+2\left(z_{\phi_{r_{2}}}-\phi_{r_{2}}\right)^{2} \\
& +3\left(z_{x_{r_{2}}}-x_{r_{2}}\right)^{2}+3\left(z_{y_{r_{2}}}-y_{r_{2}}\right)^{2} .
\end{aligned}
$$

The 1st, 2nd, 4th, and 5th equations of $\nabla F(X)=0$ can be written as

$$
M_{1} X_{1}=N_{1}
$$

where $X_{1}=\left[\begin{array}{llll}x_{r_{2}} & y_{r_{2}} & x_{r_{1}} & y_{r_{1}}\end{array}\right]^{T}$, and

$$
M_{1}=\left[\begin{array}{cccc}
2 & 0 & -1 & 0 \\
0 & 2 & 0 & -1 \\
-1 & 0 & 2 & 0 \\
0 & -1 & 0 & 2
\end{array}\right], \quad N_{1}=\left[\begin{array}{c}
z_{x_{r_{2}}}+A \\
z_{y_{r_{2}}}+B \\
z_{x_{r_{1}}}-A \\
z_{y_{r_{1}}}-B
\end{array}\right] \text {. }
$$

Then we can compute

$$
X_{1}=\left[\begin{array}{l}
x_{r_{2}} \\
y_{r_{2}} \\
x_{r_{1}} \\
y_{r_{1}}
\end{array}\right]=M_{1}^{-1} N_{1}=\left[\begin{array}{l}
\frac{1}{3} z_{x_{r_{1}}}+\frac{2}{3} z_{x_{r_{2}}}+\frac{1}{3} A \\
\frac{1}{3} z_{y_{r_{1}}}+\frac{2}{3} z_{y_{r_{2}}}+\frac{1}{3} B \\
\frac{2}{3} z_{x_{r_{1}}}+\frac{1}{3} z_{x_{r_{2}}}-\frac{1}{3} A \\
\frac{2}{3} z_{y_{r_{1}}}+\frac{1}{3} z_{y_{r_{2}}}-\frac{1}{3} B
\end{array}\right] .
$$

From the 3rd equation of $\nabla F(X)=0$, we have

$$
\phi_{r_{2}}=\frac{1}{2}\left(z_{\phi_{r_{2}}}+z_{\phi_{r_{2}}^{r_{1}}}+\phi_{r_{1}}\right)
$$

Now we have got the last 5 equations in (13).

Submit (27) and (28) into (25), and use the definition of $A, B$, we have

$$
\begin{aligned}
F(X)= & \left(z_{\phi_{r_{1}}}-\phi_{r_{1}}\right)^{2}+2\left(z_{\phi_{r_{2}}}-\phi_{r_{2}}\right)^{2} \\
& +3\left(\frac{1}{3} z_{x_{r_{1}}}-\frac{1}{3} z_{x_{r_{2}}}+\frac{1}{3} A\right)^{2} \\
& +3\left(\frac{1}{3} z_{y_{r_{1}}}-\frac{1}{3} z_{y_{r_{2}}}+\frac{1}{3} B\right)^{2} \\
= & \left(z_{\phi_{r_{1}}}-\phi_{r_{1}}\right)^{2}+\frac{1}{2}\left(-z_{\phi_{r_{2}}}+z_{\phi_{r_{2}}^{r_{1}}}+\phi_{r_{1}}\right)^{2} \\
& +\frac{1}{3}\left(z_{x_{r_{2}}}-z_{x_{r_{1}}}-A\right)^{2}+\frac{1}{3}\left(z_{y_{r_{2}}}-z_{y_{r_{1}}}-B\right)^{2} \\
= & \left(z_{\phi_{r_{1}}}-\phi_{r_{1}}\right)^{2}+\frac{1}{2}\left(-z_{\phi_{r_{2}}}+z_{\phi_{r_{2}}}+\phi_{r_{1}}\right)^{2} \\
& +\frac{1}{3}\left[A^{2}+B^{2}+\left(z_{x_{r_{2}}}-z_{x_{r_{1}}}\right)^{2}+\left(z_{y_{r_{2}}}-z_{y_{r_{1}}}\right)^{2}\right] \\
& -\frac{2}{3}\left[\begin{array}{cc}
z_{x_{r_{2}}}-z_{x_{r_{1}}} \\
z_{y_{r_{2}}}-z_{y_{r_{1}}}
\end{array}\right]^{T}\left[\begin{array}{cc}
c_{\phi_{r_{1}}} & -s_{\phi_{r_{1}}} \\
s_{\phi_{r_{1}}} & c_{\phi_{r_{1}}}
\end{array}\right]\left[\begin{array}{l}
z_{x_{r_{2}}^{r_{1}}} \\
z_{y_{r_{2}}}^{r_{1}}
\end{array}\right] .
\end{aligned}
$$

By (11) and (12), we have

$$
\begin{aligned}
& z_{x_{r_{2}}^{r_{1}}}=\left(z_{x_{r_{2}}}-z_{x_{r_{1}}}\right) c_{z_{\phi_{r_{1}}}}+\left(z_{y_{r_{2}}}-z_{y_{r_{1}}}\right) s_{z_{\phi_{r_{1}}}}+\Delta z_{x_{r_{2}}^{r_{1}}} \\
& z_{y_{r_{2}}^{r_{1}}}=-\left(z_{x_{r_{2}}}-z_{x_{r_{1}}}\right) s_{z_{\phi_{r_{1}}}}+\left(z_{y_{r_{2}}}-z_{y_{r_{1}}}\right) c_{z_{\phi_{r_{1}}}}+\Delta z_{y_{r_{2}}^{r_{1}}} \\
& z_{\phi_{r_{2}}^{r_{1}}}=z_{\phi_{r_{2}}}-z_{\phi_{r_{1}}}+\Delta z_{\phi_{r_{2}}^{r_{1}}}
\end{aligned}
$$

Submit (30) into (29), also denote

$$
\phi=\phi_{r_{1}}-z_{\phi_{r_{1}}}
$$

and notice that $A^{2}+B^{2}=z_{x_{r_{2}}^{r_{1}}}^{2}+z_{y_{r_{2}}^{r_{1}}}^{2}$, we can have

$$
\begin{aligned}
& F(X) \\
= & \phi^{2}+\frac{1}{2}\left(\phi+\Delta z_{\phi_{r_{2}}^{r_{1}}}\right)^{2}-2 d \cos \phi-2 \delta_{b} c_{\phi_{r_{1}}}+2 \delta_{a} s_{\phi_{r_{1}}}+b \\
= & \phi^{2}+\frac{1}{2}\left(\phi+\Delta z_{\phi_{r_{2}}^{r_{1}}}\right)^{2}-2(d+q) \cos \phi+2 p \sin \phi+b \\
= & \phi^{2}+\frac{1}{2}\left(\phi+\Delta z_{\phi_{r_{2}}^{r_{1}}}\right)^{2}-2 a \cos (\phi+\alpha)+b
\end{aligned}
$$

where $d, \delta_{a}, \delta_{b}, b, p, q, a, \alpha$ are given by (11) and (12).

Now we have proved that $F(X)=f(\phi)$ for all the stationary points $X$. Furthermore, it can be proved that for stationary points $X, \nabla F(X)=0$ is equivalent to $f^{\prime}(\phi)=0$, and $\nabla^{2} F(X)>0$ is equivalent to $f^{\prime \prime}(\phi)>0$. The details are omitted due to the space limit.

Thus the minimization of $F(X)$ in (7) is equivalent to minimizing $f(\phi)$ in (10). This completes the proof.

\section{B. Proof of Theorem 3}

First, note that $f(\phi)$ can be divided into two parts,

$$
f_{1}(\phi)=\phi^{2}+\frac{1}{2} \sum_{i=2}^{n+1}\left(\phi+\Delta z_{\phi_{r_{i}}^{r_{1}}}\right)^{2}
$$

and

$$
f_{2}(\phi)=-2 a \cos (\phi+\alpha)+b .
$$

Obviously, $f_{1}$ is convex and the minimum of $f_{1}$ is $\phi_{\min }=$ $-\frac{1}{n+2} \sum_{i=2}^{n+1} \Delta z_{\phi_{r_{i}}^{r_{1}}}$. Since $\Delta z_{\phi_{r_{i}}^{r_{1}}} \in[-\pi, \pi]$, we have $\phi_{\min } \in$ $\left[-\frac{n}{n+2} \pi, \frac{n}{n+2} \pi\right] \in(-\pi, \pi)$. Then $f_{1}(\phi)$ must be monotone decreasing in $(-\infty,-\pi]$ and monotone increasing in $[\pi, \infty)$.

On the other hand, $f_{2}$ is periodical and the minimal value is achieved at $-\alpha,-2 \pi-\alpha, 2 \pi-\alpha$. Since $\alpha \in[-\pi, \pi]$, we have $-2 \pi-\alpha \leq-\pi$ and $2 \pi-\alpha \geq \pi$. So $f_{1}(\phi)$ is monotone 
decreasing in $(-\infty,-2 \pi-\alpha]$ and is monotone increasing in $[2 \pi-\alpha,+\infty)$. Thus we have

$$
\begin{aligned}
& f(\phi) \geq f(2 \pi-\alpha), \quad \forall \phi \geq 2 \pi-\alpha, \\
& f(\phi) \geq f(-2 \pi-\alpha), \quad \forall \phi \leq-2 \pi-\alpha,
\end{aligned}
$$

and thus the global minimum of $f(\phi)$ is in the interval $[-2 \pi-$ $\alpha, 2 \pi-\alpha]$.

Second, denote $e=\sum_{i=2}^{n+1} \Delta z_{\phi_{r_{i}}^{r_{1}}} \in[-n \pi, n \pi]$, then

$$
f^{\prime}(\phi)=2 a \sin (\phi+\alpha)+(n+2) \phi+e
$$

and

$$
f^{\prime \prime}(\phi)=2 a \cos (\phi+\alpha)+(n+2) .
$$

If $a<1+\frac{n}{2}$, we have $f^{\prime \prime}(\phi)>0$ for any $\phi$. Thus $f(\phi)$ is convex and there is one and only one minimum.

Third, consider the case when $a \geq 1+\frac{n}{2}$. Let $f^{\prime \prime}(\phi)=0$, we get four roots $\phi_{1}, \phi_{2}, \phi_{3}, \phi_{4}$ as given in (22). Note that $\phi_{1}, \phi_{2}, \phi_{3}, \phi_{4}$ divide interval $[-2 \pi-\alpha, 2 \pi-\alpha]$ into 5 intervals where $f^{\prime}$ is monotone in each of the intervals, i.e., $[-2 \pi-$ $\left.\alpha, \phi_{1}\right),\left[\phi_{1}, \phi_{2}\right),\left[\phi_{2}, \phi_{3}\right),\left[\phi_{3}, \phi_{4}\right)$, and $\left[\phi_{4}, 2 \pi-\alpha\right]$. So the number of minima of $f(\phi)$ can be analyzed by observing the values $f^{\prime}(-2 \pi-\alpha), f^{\prime}\left(\phi_{1}\right), f^{\prime}\left(\phi_{2}\right), f^{\prime}\left(\phi_{3}\right), f^{\prime}\left(\phi_{4}\right), f^{\prime}(2 \pi-$ $\alpha)$. For example, if $f^{\prime}\left(\phi_{4}\right)<0$ and $f^{\prime}(2 \pi-\alpha)>0$, then there is a minima in interval $\left[\phi_{4}, 2 \pi-\alpha\right]$ (see Fig. 4(d)).

Note that $e \in[-n \pi, n \pi]$, so we have

$$
\begin{aligned}
f^{\prime}(-2 \pi-\alpha) & =(2+n)(-2 \pi-\alpha)+e \\
& =-(2+n)(\pi+\alpha)-(2+n) \pi+e \\
& \leq-(2+n) \pi+e \leq-2 \pi<0 \\
f^{\prime}(2 \pi-\alpha) & =(2+n)(2 \pi-\alpha)+e \\
& =(2+n)(\pi-\alpha)+(2+n) \pi+e \\
& \geq(2+n) \pi+e \geq 2 \pi>0
\end{aligned}
$$

Since $f^{\prime}\left(\phi_{1}\right) \leq 0 \Rightarrow f^{\prime}\left(\phi_{2}\right)<0, f^{\prime}\left(\phi_{2}\right) \geq 0 \Rightarrow f^{\prime}\left(\phi_{3}\right)>0$, $f^{\prime}\left(\phi_{3}\right) \leq 0 \Rightarrow f^{\prime}\left(\phi_{4}\right)<0$, there are only 8 possible cases which are listed in Table II. So there are at most three minima in $[-2 \pi-\alpha, 2 \pi-\alpha]$. This completes the proof.

\section{REFERENCES}

[1] F. Lu and E. Milios. Globally consistent range scan alignment for environment mapping. Autonomous Robots, 4(4): 333-349, 1997.

[2] U. Frese, P. Larsson, and T. Duckett. A multilevel relaxation algorithm for simultaneous localization and mapping. IEEE Trans. on Robotics, 21(2):196-207, 2005.

[3] E. Olson, J. Leonard, and S. Teller. Fast iterative optimization of pose graphs with poor initial estimates. IEEE International Conference on Robotics and Automation (ICRA), pp. 2262-2269, 2006.

[4] K. Konolige, G. Grisetti, R. Kummerle, B. Limketkai, and R. Vincent. Efficient sparse pose adjustment for 2D mapping. IEEE/RSJ International Conference on Intelligent Robots and Systems (IROS), pp. 22-29, 2010.

[5] F. Dellaert, J. Carlson, V. Ila, K. Ni, and C. E. Thorpe. Subgraph-preconditioned conjugate gradients for large scale SLAM. IEEE/RSJ International Conference on
Intelligent Robots and Systems (IROS), pp. 2566-2571, 2010.

[6] G. Grisetti, C. Stachniss, and W. Burgard. Non-linear constraint network optimization for efficient map learning. IEEE Transations on Intelligent Transportation Systems. 10(3): 428-439, 2009.

[7] X. S. Zhou and S. I. Roumeliotis. Determining the Robotto-Robot 3D relative pose using combinations of range and bearing measurements: 14 minimal problems and closed-form solutions to three of them. IEEE/RSJ International Conference on Intelligent Robots and Systems (IROS), pp. 2983-2990, 2010.

[8] S. Huang, H. Wang, U. Frese, and G. Dissanayake. On the number of local minima to the point feature based SLAM problem. IEEE International Conference on Robotics and Automation (ICRA), pp. 2074-2079, 2012.

[9] R. Kummerle, B. Steder, C. Dornhege, M. Ruhnke, G. Grisetti, C. Stachniss, and A. Kleiner. SLAM benchmarking webpage. http://kaspar.informatik.unifreiburg.de//slamEvaluation/datasets.php, 2009.

[10] L. Carlone, R. Aragues, J. Castellanos, and B. Bona. A linear approximation for graph-based simultaneous localization and mapping. Robotics: Science and Systems (RSS), 2011.

[11] K. S. Arun, T. S. Huang, and S. D. Blostein. Least squares fitting of 3-D point sets. IEEE Transactions on Pattern Analysis and Machine Intelligence, PAMI-9(5): 698-700. 1987.

[12] J. L. Martinez, J. Morales, A. Mandow, A. GarciaCerezo, Incremental closed-form solution to globally consistent 2D range scan mapping with two-step pose estimation, The 11th IEEE International Workshop on Advanced Motion Control, 21-24 March 2010, pp. 252257, 2010.

[13] D. L. Rizzini. Towards a closed-form solution of constraint networks for Maximum Likelihood mapping. The 14th International Conference on Advanced Robotics (ICAR 2009), pp. 1-5, 2009.

[14] V. Ila, J. M. Porta, and J. Andrade-Cetto. Informationbased compact pose SLAM. IEEE Transactions on Robotics, 26(1): 78-93, 2010.

[15] K. Ni, D. Steedly, and F. Dellaert. Tectonic SAM: Exact, out-of-core, submap-based SLAM. IEEE International Conference on Robotics and Automation (ICRA), pp. 1678-1685, 2007.

[16] S. Huang, Z. Wang, G. Dissanayake, and U. Frese. Iterated SLSJF: A sparse local submap joining algorithm with improved consistency. 2008 Australiasan Conference on Robotics and Automation, 2008.

[17] B. Kim, M. Kaess, L. Fletcher, J. Leonard, A. Bachrach, N. Roy, and S. Teller. Multiple relative pose graphs for robust cooperative mapping. IEEE International Conference on Robotics and Automation (ICRA), pp. 31853192, 2010. 